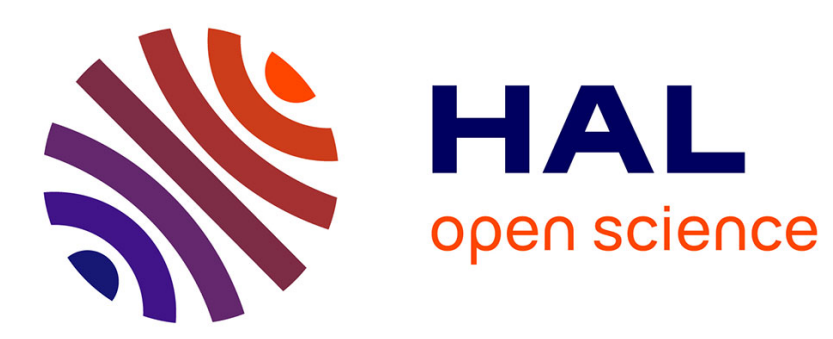

\title{
Étude et réalisation d'un amplificateur galvanométrique linéaire ou intégrateur de performances élevées
}

\author{
R. Vergne, J.L. Porteseil
}

\section{To cite this version:}

R. Vergne, J.L. Porteseil. Étude et réalisation d'un amplificateur galvanométrique linéaire ou intégrateur de performances élevées. Revue de Physique Appliquée, 1971, 6 (1), pp.95-103. 10.1051/rphysap:019710060109500 . jpa-00243507

\section{HAL Id: jpa-00243507 https://hal.science/jpa-00243507}

Submitted on 1 Jan 1971

HAL is a multi-disciplinary open access archive for the deposit and dissemination of scientific research documents, whether they are published or not. The documents may come from teaching and research institutions in France or abroad, or from public or private research centers.
L'archive ouverte pluridisciplinaire HAL, est destinée au dépôt et à la diffusion de documents scientifiques de niveau recherche, publiés ou non, émanant des établissements d'enseignement et de recherche français ou étrangers, des laboratoires publics ou privés. 


\title{
ÉTUDE ET RÉALISATION \\ D'UN AMPLIFICATEUR GALVANOMÉTRIQUE LINÉAIRE OU INTÉGRATEUR DE PERFORMANCES ÉLEVÉES
}

\author{
par R. VERGNE et J. L. PORTESEIL (*) \\ Laboratoire d'Electrostatique et de Physique du Métal (**), \\ Cedex No 166, 38, Grenoble-Gare, France \\ (Reçu le 3 juin 1970, révisé le 9 novembre 1970)
}

\begin{abstract}
Résumé. - L'étude des amplificateurs galvanométriques linéaires ou intégrateurs, considérés comme systèmes asservis, montre que leur fonction de transfert peut se mettre sous la forme d'un produit de deux termes : l'un étant le terme recherché, l'autre un terme perturbateur correspondant à un filtre passe-bas. Il est possible de corriger l'influence de ce dernier en utilisant des boucles de retour convenables. L'examen des critères de stabilité confirme que de telles corrections sont possibles et met en évidence leur limite. Pour les parfaire, on peut mettre en série avec le système corrigé un filtre ayant une fonction de transfert inverse de celle du filtre perturbateur.

Les résultats expérimentaux obtenus confirment le calcul. Ils montrent l'intérêt des amplificateurs galvanométriques linéaires dans le domaine des gains élevés. En effet, après correction, on passe sans affaiblissement et à moins de $20^{\circ}$ de déphasage : $1000 \mathrm{~Hz}$ au gain $10^{4}, 700 \mathrm{~Hz}$ au gain $10^{5}$ et $400 \mathrm{~Hz}$ au gain $10^{6}$. En ce qui concerne l'intégrateur très bas niveau, sa stabilité est exceptionnelle. Nous avons pu obtenir des dérives intrinsèques correspondant à des tensions moyennes ramenées à l'entrée de quelques dizaines de picovolts $\left(1\right.$ picovolt $\left.=10^{-12} \mathrm{~V}\right)$ pendant des durées voisines d'une heure. Même dans le cas où l'on exige la plus grande stabilité (galvanomètre lent) la bande passante de l'intégrateur reste très acceptable : moins de $20^{\circ}$ de déphasage à $1100 \mathrm{~Hz}$ et un temps de montée de $100 \mu \mathrm{s}$.

Cette étude montre qu'il est possible de corriger l'essentiel des défauts des amplificateurs galvanométriques sans en altérer les qualités.
\end{abstract}

Abstract. - The dynamical behaviour of galvanometric amplifiers is derived from servomechanism theory. It is shown that their transfer function has the form of the product of two terms, one of them being the ideal function and the other being the transfer function of a low-pass filter. It is possible to correct for the influence of this term by using suitable feedback loops. The theoretical stability conditions show that such a correction is possible to a certain amount. A further correction is performed by a tunable active network which almost cancels the effect of the lowpass filter.

The amplifier described in this paper features a good frequency response at high gains : its bandwidth values are 1000 CPS, 700 CPS and 400 CPS at gains $10^{4}, 10^{5}$ and $10^{6}$, with a phase lag less than $20^{\circ}$. The drift of the long-term integrator proved to be extremely low : during recordings up to 54 minutes, the input error voltage was less than a few $10^{-11}$ volt. Even with a slow (sensitive) galvanometer the integrator has a good frequency response : phase lag less than $20^{\circ}$ at $1100 \mathrm{CPS}$, rise time $100 \mu \mathrm{s}$.

This work shows that it is possible to improve to a great amount the behaviour of galvanometric amplifiers.

I. Introduction. - Nous nous étions initialement proposés de construire un fluxmètre de grande sensibilité. Nous désirons pouvoir étudier des variations de flux de l'ordre de $10^{-5}$ weber étalées sur des temps de mesure de 30 minutes avec des dérives intrinsèques de l'appareil n'excédant pas $1 \%$ de la valeur précédente durant ce même temps. Les f.é. m. mises en jeu sont alors de quelques nanovolts et les dérives intrinsèques tolérées correspondent à des tensions moyennes ramenées à l'entrée de quelques dizaines de picovolts.

(*) Une partie de ce travail constitue la thèse de $3^{\mathrm{e}}$ Cycle de J. L. Porteseil.

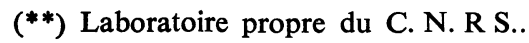

REVUE DE PHYSIQUE APPLIQUÉE. - T. 6, No 1, MARS 1971
Nous désirions de plus pouvoir mesurer des sauts de flux de très faible amplitude ; ce qui suppose que notre appareil ait une bande passante suffisante. De nombreux auteurs ont étudié des fluxmètres. On peut les classer en deux catégories : ceux qui utilisent des intégrateurs analogiques constitués par des maillons purement électroniques et ceux qui utilisent les amplificateurs galvanométriques $\left({ }^{1}\right)$. A notre connaissance, et dans l'état actuel de la technique, cette première classe d'appareils ne nous permet pas de résoudre notre problème. Ceci nous a amenés à reprendre l'étude des

(1) Remarque : Dans l'historique et dans la bibliographie nous avons seulement considéré ces derniers. 
amplificateurs galvanométriques en les considérant comme un cas particulier de systèmes asservis. Nous nous sommes attachés à améliorer leurs qualités et à corriger leurs défauts. Ce faisant, nous avons obtenu des amplificateurs linéaires pouvant travailler aux gains élevés avec une impédance d'entrée et une bande passante acceptables et un intégrateur très bas niveau d'une exceptionnelle stabilité. Ce dernier nous a permis de résoudre le problème posé.

Dans ce mémoire nous développerons d'abord les principes mis en œuvre pour résoudre le problème, nous ferons ensuite un bref historique nous permettant de situer ce travail dans son contexte et nous préciserons enfin les résultats obtenus.

II. Etude des amplificateurs galvanométriques considérés comme des systèmes asservis. - 1) "L'AMPLIFICATEUR A PHOTOCELlULes » (Fig. 1). - La tension à

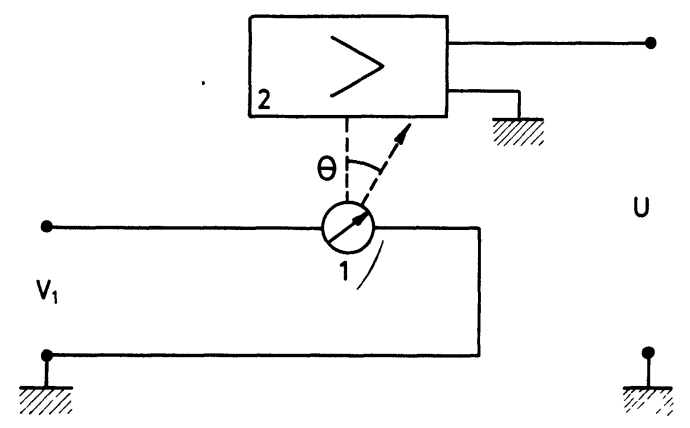

FIG. 1. - L'amplificateur à photocellules. 1 Galvanomètre, 2 Récepteur photoélectrique et étages d'amplification. étudier est appliquée aux bornes d'un galvanomètre et entraîne la déviation angulaire $\theta$ du cadre. Celle-ci se traduit par le déplacement d'un spot lumineux sur un récepteur photoélectrique différentiel. Le signal ainsi obtenu est convenablement amplifié. Il donne naissance à une tension $\mathrm{U}$ proportionnelle à $\theta$, tant que $\theta$ reste petit. C'est ce dispositif que nous appellerons « amplificateur à photocellules ». Il constitue la boucle directe du système asservi. Il semble que ce soit R. F. Edgar [1] qui l'ait imaginé le premier.

2) Notations. - Dans toute la suite de ce travail nous appellerons :

$\Gamma$ la constante de torsion de la suspension du galvanomètre,

$f$ l'amortissement mécanique du cadre,

$I$ son moment d'inertie,

$\rho$ la résistance électrique du cadre,

$\lambda$ son inductance,

$\varphi_{0}$ le flux dans l'entrefer,

$\theta$ la déviation angulaire du cadre,

$V_{1}$ la tension aux bornes du galvanomètre,

$i_{1}$ l'intensité le parcourant,

$U$ la tension de sortie de l' «amplificateur à photocellules $»$

3) SA FONCTION DE TRANSFERT. - Dans l'hypothèse où le récepteur photoélectrique différentiel et les étages d'amplification qui le suivent n'introduisent pas de déphasage appréciable la fonction de transfert de l'amplificateur s'écrit :

$$
\tau(p)=\frac{U}{V_{1}}=\frac{\varphi_{0} G}{\lambda I p^{3}+(f \lambda+\rho I) p^{2}+\left(f \rho+\lambda \Gamma+\varphi_{0}^{2}\right) p+\Gamma \rho}
$$

où, $G$ est le gain angulaire défini par $G=U / \theta$.

Le système est un filtre passe-bas du $3^{\mathrm{e}}$ ordre dont les caractéristiques sont déterminées par le galvanomètre. Sa bande passante est très étroite et son impédance d'entrée faible. Par contre, son gain statique $K_{0}^{\prime}=\varphi_{0} G / \Gamma \rho$ est très élevé et peut atteindre $10^{9}$ à $10^{10}$; c'est là le principal intérêt du dispositif. L' ' amplificateur à photocellules » constitue la boucle directe du système asservi.

4) INTRODUCTION D'UNE BOUCLE DE RETOUR. Expression de la fonction de transfert du système

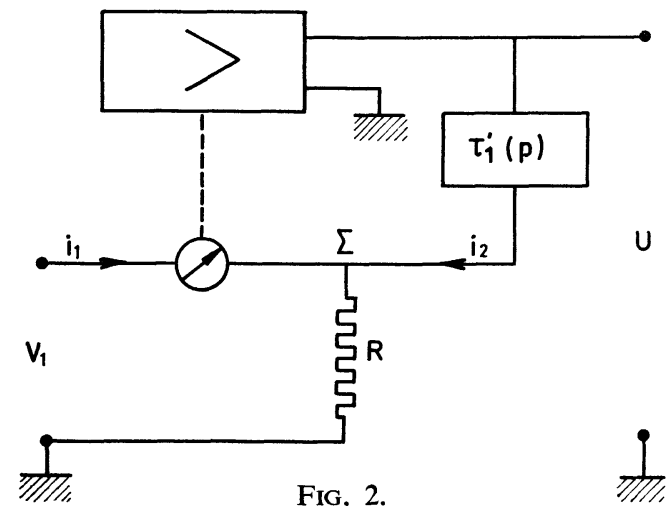

asservi (Fig. 2 et 3). Correction des défauts dus au galvanomètre (Fig. 4).

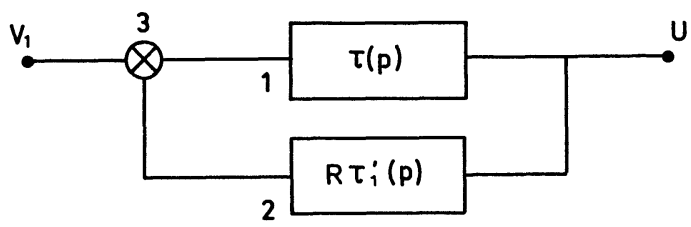

FIG. 3. -1 boucle directe; 2 boucle de retour; 3 élément comparateur.

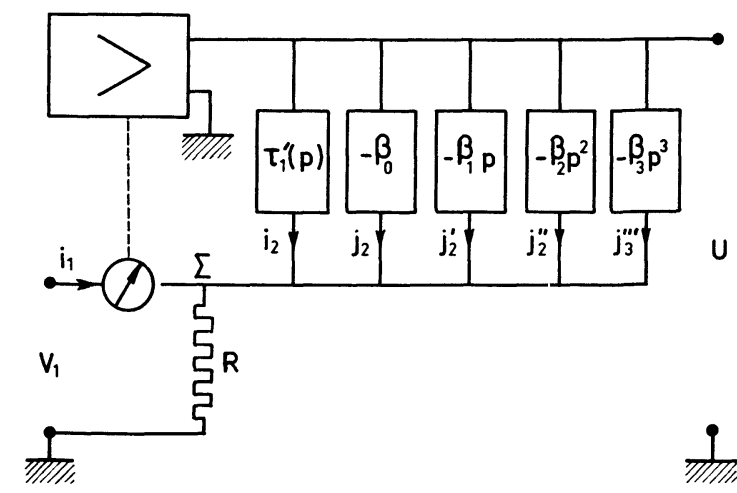

FIG. 4. - Schéma général des diverses boucles de retour. 
Le galvanomètre est soumis aux deux tensions $V_{1}$ et $V_{1}^{\prime}$ qui sont en opposition. $V_{1}^{\prime}=R i_{2}=R \tau_{1}^{\prime}(p) U$. Nous avons réalisé un système asservi dont le galvanomètre est l'élément comparateur. Sa fonction de transfert $W(p)$ s'écrit :

Si le gain en boucle couverte $K_{0}$ était infini, les caractéristiques propres du galvanomètre n'interviendraient pas et $W(p) \sim 1 / \tau_{1}^{\prime}(p)$. Mais il est impossible d'augmen-

$$
\begin{aligned}
& w(p)=\frac{\tau(p)}{1+\tau(p) \tau^{\prime}(p)} \\
& w(p)=\frac{1}{\frac{1}{K_{0}}\left(A p^{3}+B p^{2}+C^{\prime} p+1\right)+R \tau_{1}^{\prime}(p)}
\end{aligned}
$$

ter $K_{0}$ au-delà d'une certaine limite, ne serait-ce que pour des raisons de stabilité et de bruit. Ceci nous amène à essayer de corriger les défauts du galvanomètre en introduisant, en plus de la boucle de retour principale, d'autres boucles de retour destinées à diminuer les termes en $p^{3}, p^{2}, p$ et $p^{0}$. Dans le cas le plus général, nous réaliserons 4 boucles de retour (Fig. 4) fournissant des courants $j_{2} ; j_{2}^{\prime} ; j_{2}^{\prime \prime} ; j_{2}^{\prime \prime \prime}$ tels que nous ayons :

$$
\begin{gathered}
j_{2}=-\beta_{0} U ; j_{2}^{\prime}=-\beta_{1} p U ; j_{2}^{\prime \prime}=-\beta_{2} p^{2} U ; \\
j_{2}^{\prime \prime \prime}=-\beta_{3} p^{3} U .
\end{gathered}
$$

La fonction de transfert d'un tel système s'écrit alors :

$$
w(p)=\frac{1}{\frac{1}{K_{0}}\left[\left(A-K_{0} R \beta_{3}\right) p^{3}+\left(B-K_{0} R \beta_{2}\right) p^{2}+\left(C^{\prime}-K_{0} R \beta_{1}\right) p+\left(1-K_{0} R \beta_{0}\right)\right]+R \tau_{1}^{\prime}(p)}
$$

Le dispositif ainsi réalisé est l'équivalent d'un amplificateur linéaire idéal mis en série avec un filtre passebas du troisième ordre. Pour les fréquences pas trop élevées, ce dernier est assimilable à un filtre du second ordre. Sa fréquence propre est bien plus élevée que celle du galvanomè̀re : $\beta \gg 1$; mais il est très peu amorti, $C^{\prime} / \beta$ étant $\mathrm{p}$ stit. Pour réaliser un amplificateur linéaire acceptable il faut l'amortir, augmenter sa fréquence propre et diminuer l'influence du terme en $p^{2}$. Pour des fréquences plus élevées, il devient nécessaire de réduire également le terme en $p^{3}$.

On arrive à ce résultat en utilisant 4 boucles de retour : une boucle principale et 3 correctives fournissant des tensions $V^{\prime}$ en opposition avec $V_{1}$ telles que :

$$
\begin{gathered}
V_{1}^{\prime}=\frac{R}{R^{\prime}} U ; V_{2}^{\prime} \sim R p U ; V_{3}^{\prime} \sim-R p^{2} U ; \\
V_{4}^{\prime} \sim-R p^{3} U .
\end{gathered}
$$

La fonction de transfert s'écrit alors :

où $W_{0}(p)=k$ et où $\beta=K_{6} / k$ n'est autre que le gain de boucle.

$$
w(p)=w_{0}(p) \frac{1}{\left(a-\alpha_{3}\right) p^{3}+\left(b-\alpha_{2}\right) p^{2}+\left(C^{\prime}+\alpha_{1}\right) p+\left(1+\alpha_{0}\right)}
$$

$a, b, c$ étant des constantes dépendant du galvanomètre et $\alpha_{0}, \alpha_{1}, \alpha_{2}, \alpha_{3}$ les taux de réinjection des termes en $p^{0}, p^{1}, p^{2}$ et $p^{3}$.

b) Un amplificateur intégrateur peut ètre considéré comme un amplificateur linéaire très amorti dont le gain tend vers l'infini pour $\omega=0$. Dès lors, on voit qu'il suffit de supprimer la résistance $R^{\prime}$ et d'augmenter le terme en $p$.

La fonction de transfert devient :

$$
w(p)=\frac{1}{\theta p} \frac{1}{\frac{1}{K_{0} \theta}\left(A p^{2}+B p+C^{\prime}+\frac{1}{p}\right)+1}
$$

où $\theta$ est la constante de temps de l'intégrateur.

$W_{0}(p)=1 / \theta p$ est la fonction de transfert de l'intégrateur idéal. L'expression précédente fait apparaitre 2 types de défauts: des défauts à très basse fréquence dus au terme en $1 / p$ et des défauts à haute fréquence dus aux termes en $p^{2}$ et $p$. Le premier type de défauts est dû au couple de rappel du galvanomètre, il est possible de le corriger rigoureusement par une boucle de retour convenable: $V_{2}^{\prime} \sim-R p^{0} U$. Cette correction étant faite, on a :

$$
w(p)=w_{0}(p) \frac{1}{a^{\prime} p^{2 \mathbf{2}}+b^{\prime \prime} p+c^{\prime}}
$$


autrement dit un intégrateur idéal mis en série avec un filtre passe-bas du second ordre. Pour corriger les défauts de celui-ci il suffit d'introduire les boucles précédentes en $-p^{2}$ et $-p^{3}$. On obtient finalement;

$$
w(p)=w_{0}(p) \frac{1}{\left(a^{\prime}-\alpha_{2}^{\prime}\right) p^{2}+\left(b^{\prime}-\alpha_{1}^{\prime}\right) p+c^{\prime}}
$$

en utilisant des notations analogues à celles utilisées pour l'amplificateur linéaire. Le schéma de principe de l'appareil est celui de la figure 5 .

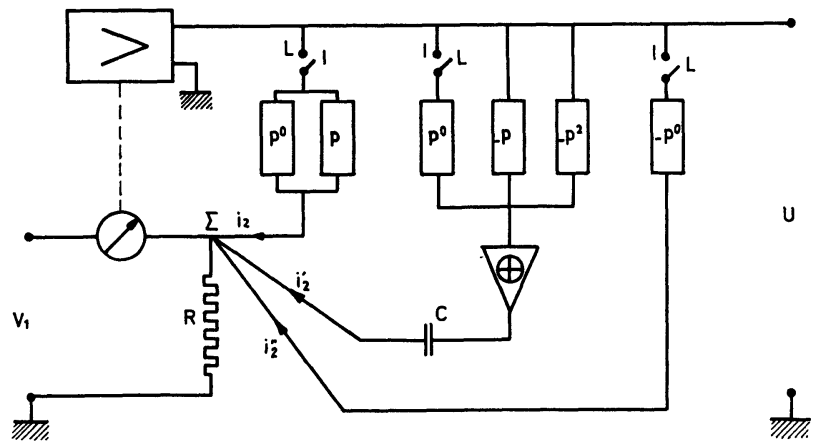

Fig. 5. - Schéma synoptique général de l'amplificateur linéaire et intégrateur.

Nous voyons que si nous pouvons faire ces corrections, toutes les caractéristiques du galvanomètre disparaissent de la fonction de transfert. Seules interviennent alors la sensibilité et la stabilité. La première permet d'obtenir un grand gain en boucle ouverte, la seconde est primordiale dans l'utilisation en intégrateur. L'étude de la stabilité du système en fonction des taux de réinjection montre qu'une correction partielle est possible ; mais qu'une correction totale est impossible sauf pour la compensation du couple de rappel. Ce résultat est intuitif physiquement. Nous verrons cependant que le gain de performances obtenu par ce procédé est très appréciable.

6) Correction extérieure. - Pour parfaire nos résultats, nous avons été amenés à développer un autre type de correction que nous avons appelée extérieure car elle ne nécessite qu'une connaissance des caractéristiques globales du système bouclé et non de ses mécanismes propres.

Nous avons montré que la fonction de transfert du système se présente toujours sous la forme :

$$
w(p)=w_{0}(p) w_{1}(p)
$$

$w_{0}(p)$ étant la fonction idéale recherchée et $w_{1}(p)$ une fonction parasite limitant les performances. Si nous savons faire un filtre de fonction de transfert $\frac{1}{w_{1} p}$ il nous suffira de le mettre en série avec le système précédent pour obtenir le résultat recherché. Le problème à résoudre est donc le suivant : réaliser un filtre passe-haut de troisième ordre ayant une fonction de transfert inverse de celle du système. C'est un problème que l'on ne sait pas résoudre dans le cas le plus général. Une étude plus approfondie montre qu'on peut le résoudre avec une approximation suffisante en mettant en série deux filtres passe-haut, l'un du second ordre, l'autre du premier ordre. On sait réaliser de tels filtres. L'étude de la stabilité du système montre qu'une correction de ce type ne peut être que partielle. L'ensemble de ces deux corrections a donné dans la pratique d'excellents résultats.

III. Historique. - L'amplification de faibles tensions électriques est un problème que de nombreux auteurs ont cherché à résoudre en utilisant un amplificateur galvanométrique. Ce type d'appareil a reçu divers perfectionnements successifs. Un rapide historique nous permettra de préciser l'évolution de la question et de replacer le présent travail dans son contexte.

L'amplificateur galvanométrique a été proposé tout d'abord pour étudier des phénomènes magnétiques. Le montage intégrateur est particulièrement intéressant car il permet de mesurer une variation de flux en intégrant la tension électrique qui en résulte, à partir de la loi de Faraday $e=-\mathrm{d} \varphi / \mathrm{d} t$. De nombreux auteurs ont étudié ce montage en vue de réaliser un fluxmètre. Ce sont, par ordre chronologique :

R. F. Edgar, en 1937 [1], R. H. Dicke, en 1948 [2], P. P. Cioffi, en 1950, puis en 1954 [3], [4], R. I. Berge et C. A. Guderjahn, en 1954 [5], S. P. Kapitza, en 1955 [6], P. Lerond et $\AA$. Thulin, en 1959 [7], R. R. Bockemuehl et P. Wood, en 1960 [8].

Tous ces auteurs emploient un réseau donnant une contre-réaction proportionnelle à la fréquence qu'ils réalisent soit à l'aide d'une capacité, soit à l'aide d'une mutuelle inductance. Les appareils décrits en 1959 et postérieurement comprennent en outre un réseau de réaction destiné à compenser la constante de rappel du galvanomètre.

L'étude de S. P. Kapitza [6] présente un intérêt particulier. Cet auteur analyse le fonctionnement de l'intégrateur et essaie d'augmenter sa bande passante. Il introduit une correction dans la boucle directe destinée à compenser les défauts du galvanomètre considéré comme un filtre passe-bas du second ordre. Pour cela il insère dans la chaîne d'amplification un filtre passif ayant une fonction de transfert inverse de celle $\mathrm{du}$ galvanomètre. Il réalise ce filtre passe-haut à l'aide d'une résistance, d'une self et d'une capacité montées en série. Malgré son intérêt théorique, ce procédé est limité en pratique par les valeurs trop élevées qu'il faudrait donner à la self. Il permet d'obtenir une correction efficace aux très basses fréquences. Par contre, aux fréquences moyennes ou élevées, la correction est imparfaite ou inexistante. Toutefois, l'intégrateur décrit par Kapitza a une bande passante de plusieurs dizaines de hertz.

D'autres auteurs ne se sont pas bornés au cas de l'intégrateur et ont fait des études plus générales. Citons tout d'abord P. Grivet, M. Sauzade et R. Stefant qui reprennent le problème en 1961 [9] en utilisant comme 
détecteur photoélectrique une cellule double au sulfure de cadmium. Ce procédé simplifie notablement la construction de l'appareil mais en contrepartie limite sérieusement la réponse en fréquence car la cellule utilisée manque de rapidité (bande passante de l'ordre de 60 hertz). L'appareil ainsi réalisé fonctionne, soit en amplificateur linéaire, soit en intégrateur. Les boucles de contre-réaction résistive et capacitive, ainsi que la correction du couple de rappel sont décrites en détail.

Ces auteurs donnent pour deux de leurs réalisations les caractéristiques suivantes : Amplificateur linéaire : gain $10^{6}$, bande passante $0-20$ hertz, résistance d'entrée $5000 \Omega$; amplificateur intégrateur : sensibilité $10^{-6}$ Weber par volt de sortie, plus petite variation de flux mesurable $10^{-7}$ weber, dérive $3 \times 10^{-7}$ weber par minute. Dans les deux cas, ces auteurs utilisent un galvanomètre ST 5BD de S. E. F. R. A. M., de période propre $T_{0}=14 \mathrm{~s}$.

En 1963, J. Max et H. Chevalier [10] réalisent un amplificateur à courant continu ayant une bande passante de 20 hertz. Ils étudient la stabilité du fonctionnement et l'origine des dérives de l'appareil. Simultanément et sur des bases analogues, B. Cazenave [11] conduit une étude qui aboutit à l' « Amplispot », de S. E. F. R. A. M. réalisation commerciale du dispositif. Cet appareil a une bande passante de quelques dizaines de hertz et peut fonctionner soit en amplificateur linéaire, soit en intégrateur.

$\mathrm{Au}$ Laboratoire P. Brissonneau et R. Péchart [12] ont étudié un fluxmètre analogue dans ses principes, à celui décrit par $\mathrm{S}$. Kapitza. Ils ont obtenu des résultats comparables à ceux de M. Sauzade avec cependant des dérives légèrement plus faibles. Par la suite, P. Brissonneau [12] a repris cette étude en y ajoutant de notables améliorations, nous nous sommes inspirés de ses idées en ce qui concerne la partie optique et mécanique de notre montage. A la même époque nous avons fait une étude critique très détaillée de l' « Amplispot » de manière à voir dans quelle mesure il était possible de l'améliorer. Nous sommes arrivés à la conclusion qu'il était nécessaire de lui adjoindre un réseau correcteur. Nous avons soumis le problème à $\mathrm{J}$. Max qui nous a proposé le principe que nous avons développé dans le correcteur extérieur.

Dans le présent travail, nous avons essayé d'améliorer systématiquement les possibilités de l'amplificateur galvanométrique. Nous avons d'une part apporté les perfectionnements suivants par rapport aux réalisations déjà existantes : augmentation substantielle du gain en boucle ouverte; suppression pratiquement totale des déphasages autres que ceux dus au galvanomètre; diminution des bruits et des dérives de l'appareil par l'utilisation de précautions adéquates. Nous avons d'autre part apporté une contribution originale en employant des filtres actifs pour corriger les défauts du système. Les réseaux actifs nous ont permis : de réaliser des corrections d'ordre supérieur (termes en $p^{2}$ et $p^{3}$ ); de modifier le principe de correction du couple de rappel du galvanomètre, et par là même d'éliminer certains inconvénients ; enfin, de réaliser un réseau correcteur extérieur d'emploi commode.

VI. Les résultats expérimentaux. - Nous consacrerons d'abord un paragraphe à l'amplificateur à photocellules, puis nous étudierons successivement l'amplificateur linéaire et intégrateur et nous montrerons enfin l'amélioration des performances consécutive à la correction extérieure.

1) LES CARACTÉRISTIQUES DE L'AMPLIFICATEUR A PHOTOCELLULES. - a) Le gain en boucle ouverte. La qualité primordiale de l'amplificateur à photocellules réside dans un très grand gain. Nous avons défini le gain angulaire par la relation : $U=G \theta$; $\theta$ étant la rotation du cadre. Par la suite nous rapporterons ce gain à $\theta^{\prime}$ c'est-à-dire à la rotation du rayon lumineux $U=G^{\prime} \theta^{\prime}=G^{\prime} g \theta$; nous rapporterons également la sensibilité en courant à $\theta^{\prime}$; soit

$$
\sigma^{\prime}=g \sigma=\frac{g \varphi_{0}}{\Gamma}
$$

avec $g=2$ ou 4 suivant que le galvanomètre est à simple ou double réflexion. Le gain angulaire $G^{\prime}$ peut être de l'ordre de $10^{6}$ volt. $\mathrm{rad}^{-1}$. Dans ces conditions, la pleine échelle de l'appareil soit \pm 10 volts est obtenue pour des déplacements du spot lumineux de $\pm 3 \mu$ environ. Le galvanomètre travaille toujours très près de sa position de zéro ce qui justifie $a$ posteriori l'hypothèse de linéarité du système dans sa zone de fonctionnement. A titre de comparaison, la version commerciale de S. E. F. R. A. M. l' " Amplispot » fonctionne avec un gain angulaire

$$
G^{\prime} \sim 10^{4} \text { volt } \cdot \mathrm{rad}^{-1} \text {. }
$$

Le gain en tension du système

$$
K_{0}=\frac{G^{\prime} \sigma^{\prime}}{R_{t}}=\frac{G \sigma}{R_{t}}
$$

dépend de la résistance $R_{t}$ du circuit galvanométrique. Tous nos essais ont été réalisés avec les deux galvanomètres dont les caractéristiques sont données dans le tableau I. Le galvanomètre (1) est du type à cadre immergé à double réflexion (SV 4SD de S.E.F. R. A.M.) le galvanomètre (2) est du type oscillographe, moins

\begin{tabular}{|c|c|c|c|}
\hline Grandeur & Unité & (1) & (2) \\
\hline Sensibilité $\sigma^{\prime}$ & $\operatorname{Rad} . A^{-1}$ & $2 \times 10^{5}$ & $2,2 \times 10^{4}$ \\
\hline Résistance interne & $\Omega$ & 21 & 100 \\
\hline Fréquence propre & $\mathrm{Hz}$ & 0,7 & 15,5 \\
\hline ans l'entrefer & weber & $2 \times 10^{-3}$ & $4,6 \times 10^{-4}$ \\
\hline de torsion & N.m.rad-1 & $3,35 \times 10^{-8}$ & $2,1 \times 10^{-8}$ \\
\hline Moment d'inertie & $\mathrm{kg} \cdot \mathrm{m}^{2}$ & $1,7 \times 10^{-9}$ & $2,2 \times 10^{-12}$ \\
\hline
\end{tabular}
sensible mais plus rapide (type E 328 de S. E.F.R.A. M.).

\section{TABLEAU I}

Le gain en tension $K_{0}$ peut atteindre $10^{10}$. 
b) Le bruit du système. - Bien que le système ne soit pas utilisable tel quel, il est intéressant de préciser les origines du bruit et d'en étudier la composition spectrale.

Les origines sont à la fois mécaniques et électriques. Parmi les premières, il y a d'une part les vibrations mécaniques transmises par le support et par l'air, d'autre part le bombardement du cadre par les molécules. Les vibrations du sol proviennent de l'agitation microsismique [13] et de diverses causes locales. Le bruit qui en résulte correspond à la fraction de cellesci passant à travers les deux filtres passe-bas constitués par le support antivibratoire et le galvanomètre luimême. Il est difficile de les chiffrer a priori ; mais on peut avoir une idée de leur importance ainsi que de celle des vibrations transmises par l'air en remplaçant le galvanomètre par un système optique fixe de mêmes caractéristiques. L'expérience montre que ce n'est pas là la cause prépondérante du bruit. Par contre on peut estimer l'ordre de grandeur des rotations aléatoires du cadre dues au mouvement brownien à partir de l'expression $\Gamma \overline{\theta^{2}}=k T$. Le bruit d'origine électrique est dû aux amplificateurs intermédiaires et à divers parasites rayonnés, en particulier à la fréquence du secteur. Le bruit de l'électronique intermédiaire est connu; il nous est donné par le constructeur. L'expérience montre qu'au gain angulaire $10^{6}$ le bruit peut être de l'ordre de quelques volts crête à crête. La valeur efficace $\sqrt{\bar{\theta}^{2}}$ calculée à la température ambiante pour le galvanomètre (1) vaut : $=0,36 \times 10^{-6}$ radian. Celle de la déviation correspondante est $0,4 \mu$. Bien qu'il n'y ait pas de relation précise entre la valeur efficace de ce phénomène aléatoire et son amplitude crête à crête, on est cependant en droit de penser qu'il est à l'origine de la majorité du bruit observé (cf. paragraphe précédent : $a$ ). Dès lors il est illusoire de vouloir l'améliorer. L'ensemble des bruits d'origine mécanique aura un spectre limité au voisinage de la fréquence propre du galvanomètre; par contre les bruits d'origine électrique auront un spectre continu allant assez loin en fréquence. A cela pourra se superposer un spectre de raies dues à des parasites locaux électriques ou mécaniques.

Pour déterminer le spectre de densité de puissance de bruit nous avons pris la transformée de Fourier de la fonction d'autocorrélation du système. Les résultats obtenus confirment pleinement les prévisions précédentes.
2) L'AMPLIFICATEUR LINÉAIRE. - Nous étudierons successivement la réponse en fréquence, l'impédance d'entrée et le bruit de l'amplificateur pour chacun des galvanomètres employés.

a) La réponse en fréquence du système. - Dans chaque cas étudié, nous avons relevé des courbes de réponse de l'amplificateur: gain et phase en fonction de la fréquence. Pour pouvoir représenter systématiquequement les résultats obtenus nous avons défini 3 fréquences $f_{0}, f_{1}$ et $f_{2}$. L'intervalle de fréquence $0-f_{0}$ représente la bande passante à \pm 6 décibels. En fait, la courbe de gain est plate (0 décibel) dans toute la partie basse fréquence du diagramme. Une erreur sur l'amplitude ne commence à apparaître qu'au voisinage de la fréquence propre du système. Quand la fréquence $f_{1}$ est atteinte, le déphasage vaut $90^{\circ}$ (quadrature retard); alors qu'il est seulement de $20^{\circ}$ pour la fréquence $f_{2}$. Cette dernière valeur du déphasage a été choisie arbitrairement. Le tableau II donne les résultats obtenus avec les 2 galvanomètres (1) et (2), pour les gains $10^{4}, 10^{5}$ et $10^{6}$. Il montre que l'amplificateur peut traiter correctement des signaux rapides.

La figure 6 représente les courbes de l'amplificateur au gain $10^{4}$ pour chacun des deux galvanomètres. Pour montrer l'intérêt qu'il y a à augmenter le gain en boucle ouverte de manière à pouvoir travailler avec les gains de boucle aussi élevés que possible, nous avons étudié l'amplificateur au gain $G^{\prime}=10^{4}$. Cette valeur est celle adoptée sur l'« Amplispot ». Le tableau III résume les résultats obtenus dans le cas du galvanomètre (2).

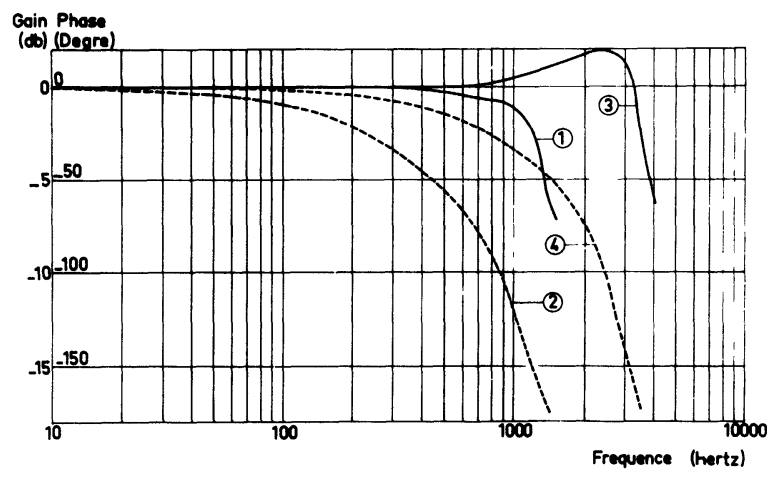

FIG. 6. - La réponse en fréquence de l'amplificateur linéaire au gain $10^{4}$. Courbe de gain et de phase en fonction de la fréquence : les courbes 1 et 2 sont relatives au galvanomètre (1), les courbes 3 et 4 au galvanomètre (2).

\section{TABLEAU II}

La partie gauche de chaque colonne est relative au galvanomètre (1), la partie droite au galvanomètre (2)

\begin{tabular}{|c|c|c|c|c|c|}
\hline$\underset{k}{\text { Gain }}$ & $\begin{array}{l}\text { Gain } \\
\text { en boucle ouverte } K_{0}\end{array}$ & $\begin{array}{l}f_{0} \\
(\mathrm{~Hz})\end{array}$ & $\begin{array}{l}f_{1} \\
(\mathrm{~Hz})\end{array}$ & $\begin{array}{l}f_{2} \\
(\mathrm{~Hz})\end{array}$ & $\underset{\mathrm{de}}{\text { Temps }} \underset{\mathrm{ms}}{\mathrm{ms}}$ \\
\hline & - (2) & (1) (2) & (1) (2) & (1) ${ }^{-}(2)$ & (1) (2) \\
\hline $10^{4}$ & $5,9 \times 10^{9} 1,6 \times 10^{8}$ & 14003980 & 7802225 & 160510 & $0,5 \quad 0,2$ \\
\hline $10^{5}$ & $5,9 \times 10^{9} 2,1 \times 10^{8}$ & 2852450 & 1871160 & 45210 & 0,5 \\
\hline $10^{6}$ & $5,9 \times 10^{9} 2,6 \times 10^{8}$ & 100650 & $79 \quad 380$ & 14116 & $5 \quad 1,5$ \\
\hline
\end{tabular}


TABLEAU III

\begin{tabular}{|c|c|c|c|c|c|}
\hline Gain & $\begin{array}{c}\text { Gain } \\
\text { en boucle } \\
\text { ouverte }\end{array}$ & $\begin{array}{c}f_{0} \\
(\mathrm{~Hz})\end{array}$ & $\begin{array}{c}f_{1} \\
(\mathrm{~Hz})\end{array}$ & $\begin{array}{c}f_{2} \\
(\mathrm{~Hz})\end{array}$ & $\begin{array}{c}\text { Temps } \\
\text { de montée } \\
\text { ms }\end{array}$ \\
\hline \multirow[t]{2}{*}{$10^{4}$} & $1,6 \times 10^{8}$ & 3980 & $2 \overline{225}$ & 510 & 0,2 \\
\hline & $10^{6}$ & 325 & 225 & 57 & 2 \\
\hline \multirow[t]{2}{*}{$10^{5}$} & $2,1 \times 10^{8}$ & 2450 & 1160 & 210 & 0,5 \\
\hline & $10^{6}$ & 85 & 67 & 15 & 8 \\
\hline \multirow[t]{2}{*}{$10^{6}$} & $2,6 \times 10^{8}$ & 650 & 380 & 116 & 1,5 \\
\hline & $10^{6}$ & 35 & 33 & 10 & 20 \\
\hline
\end{tabular}

b) L'impédance d'entrée de l'amplificateur linéaire. - Nous définissons l'impédance d'entrée à partir de la relation $Z_{i}=V_{1} / i_{1}$. Elle a pour expression :

$$
\begin{aligned}
& Z_{i}=\frac{b}{I p^{2}+f p+\Gamma}\left\{\frac{b}{\lambda I} p^{3}+\left(\frac{\lambda f}{b}+I\right) p^{2}+\right. \\
& \left.\left(f+\frac{\lambda \Gamma}{b}+\frac{\Phi_{0}^{2}}{b}+\frac{\Phi_{0} G}{b} R C\right) p+\Gamma \frac{\Phi_{0} G}{b} \frac{R}{R^{\prime}}\right\} .
\end{aligned}
$$

L'inductance du cadre du galvanomètre étant très faible, on peut en général négliger les termes contenant $\lambda$.

On peut également négliger l'amortissement électromagnétique devant celui dû à la contre-réaction. On obtient alors :

$$
Z_{i}=b+\Phi_{0} G \frac{R C p+\frac{R}{R^{\prime}}}{I p^{2}+f p+\Gamma}
$$

Cette expression devient en régime sinusoïdal de pulsation $\omega$ :

$$
Z_{i}=b+\Phi_{0} G \frac{j R C \omega+\frac{R}{R^{\prime}}}{-I \omega^{2}+j f \omega+\Gamma}
$$

A la fréquence zéro $Z_{i}$ est réelle et a pour expression: $Z_{i}=b\left(1+K_{0} / k\right) ; K_{0} / k$ étant le gain de boucle. Le tableau IV donne les valeurs de l'impédance d'entrée

\begin{tabular}{|c|c|c|c|c|}
\hline \multirow{2}{*}{ Gain } & \multicolumn{2}{|c|}{$\begin{array}{c}\text { Gain } \\
\text { en boucle ouverte }\end{array}$} & \multicolumn{2}{|c|}{$\begin{array}{l}\text { Impédance d'entrée } \\
\text { en continu (ohms) }\end{array}$} \\
\hline & (1) & (2) & (1) & (2) \\
\hline $10^{4}$ & $5,9 \times 10^{9}$ & $1,6 \times 10^{8}$ & $2,4 \times 10^{7}$ & $1,9 \times 10^{6}$ \\
\hline $10^{5}$ & $5,9 \times 10^{9}$ & $2,1 \times 10^{8}$ & $2,4 \times 10^{6}$ & $2,5 \times 10^{5}$ \\
\hline $10^{6}$ & $5,9 \times 10^{9}$ & $2,6 \times 10^{8}$ & $2,4 \times 10^{5}$ & $3,1 \times 10^{4}$ \\
\hline
\end{tabular}
pour le continu aux gains $10^{4}, 10^{5}, 10^{6}$, pour les deux galvanomètres.

\section{TABLEAU IV}

Le module de l'impédance d'entrée passe par un maximum pour une fréquence voisine de la fréquence de résonance mécanique du galvanomètre et tend vers la valeur $b$ aux fréquences élevées pour lesquelles le système n'est plus contre-réactionné.

c) Le bruit de l'amplificateur linéaire. - Le bruit du système bouclé est très différent du bruit en boucle ouverte et dépend essentiellement des conditions d'utilisation. En effet, il varie considérablement avec le gain, la bande passante et l'amplification inter-

\begin{tabular}{|c|c|c|}
\hline Gain & $10^{4}$ & $10^{5}$ \\
\hline Bruit ( $\mu \mathrm{V}$ crête à crête) & 50 & 5 \\
\hline
\end{tabular}
médiaire. Le tableau $\mathrm{V}$ donne les valeurs maximales du bruit, ramenées à l'entrée, en fonction du gain.
TABLEAU V

Ces valeurs ont été relevées dans les cas les plus défavorables où l'emploi d'une amplification intermédiaire élevée était nécessaire. Le cas se présente quand on veut obtenir un très grand gain en boucle ouverte avec le galvanomètre (2). En pratique les niveaux de bruit observés sont le plus souvent nettement inférieurs. Nous n'avons pas fait une étude systématique du bruit de l'amplificateur linéaire car nous ne disposions pas d'un corrélateur travaillant à des fréquences suffisantes.

3) L'AMPLIFICATEUR INTÉGRATEUR. - a) Stabilité de l'intégrateur. - La qualité essentielle d'un intégrateur est sa stabilité. Nous ne ferons pas ici l'analyse des causes de dérive ni de la façon de les diminuer; ce qui nous conduirait à un exposé long et fastidieux. Nous nous bornerons à en rappeler les causes principales : f. é. $m$. parasites dans le galvanomètre; f.é. $m$. parasites dans les circuits d'entrée à bas niveau, dérives mécaniques (galvanomètre, dilatation du bâti) et enfin l'électronique constituant les étages d'amplification intermédiaire et les divers filtres. Leur élimination, qui met en jeu des principes de physique très élémentaires, est une affaire de soin et de patience.

Tous les résultats qui suivent ont été obtenus après avoir réglé très soigneusement la boucle de réaction proportionnelle. Pour donner une représentation simple de la dérive, nous supposerons qu'elle a été causée par une f. é. m. constante $\bar{e}_{\mathrm{m}}$, présente aux bornes de l'intégrateur pendant toute la durée de l'essai.

\begin{tabular}{|c|c|c|c|}
\hline \multicolumn{2}{|c|}{ Galvanomètre (1) } & \multicolumn{2}{|c|}{ Galvanomètre (2) } \\
\hline & Tension moyenne & & Tension moyenne \\
\hline $\begin{array}{l}\text { Durée de } \\
\text { l'essai } \\
\text { (mn) }\end{array}$ & $\begin{array}{l}\text { ramenée } \\
\text { à l'entrée } \\
\text { (V) }\end{array}$ & $\begin{array}{c}\text { Durée de } \\
\text { l'essai } \\
\text { (mn) }\end{array}$ & $\begin{array}{l}\text { ramenée } \\
\text { à l'entrée } \\
\text { (V) }\end{array}$ \\
\hline 10 & $\pm 1,7 \times 10^{-11}$ & 10 & $10^{-10}$ \\
\hline 16 & $\pm 3,1 \times 10^{-11}$ & 10 & $\pm 8,3 \times 10^{-11}$ \\
\hline مי & $\pm 4,2 \times 10^{-11}$ & 13 & $\pm 9 \times 10^{-11}$ \\
\hline 54 & $\pm 3,4 \times 10^{-11}$ & & \\
\hline
\end{tabular}
Le tableau VI donne quelques résultats obtenus avec les galvanomètres (1) et (2).

\section{Tableau VI}

b) La réponse en fréquence de l'intégrateur (Fig. 7). - Pour relever expérimentalement les courbes de réponse de l'intégrateur, il est commode de dériver une fois un signal sinusoïdal connu. On obtient ainsi à la sortie de l'intégrateur, à une constante près, les courbes caractéristiques du filtre passe-bas mis en série avec l'intégrateur idéal. Ces courbes représentent l'écart à la perfection de l'intégrateur. C'est pourquoi nous parlerons de "bande passante » de l'intégrateur, et nous représenterons son comportement par la donnée des mêmes fréquences $f_{0}, f_{1}$ et $f_{2}$ que dans le cas de l'amplificateur linéaire. Il est également intéressant d'indiquer le temps de montée de l'échelon de tension 
TABLEAU VII

Gain en boucle ouverte $G \varphi_{0} / \Gamma b$

$(1)$
$1,8 \times 10^{9}$
$3,3 \times 10^{7}$

Goin Phose
(db) (Dagre)

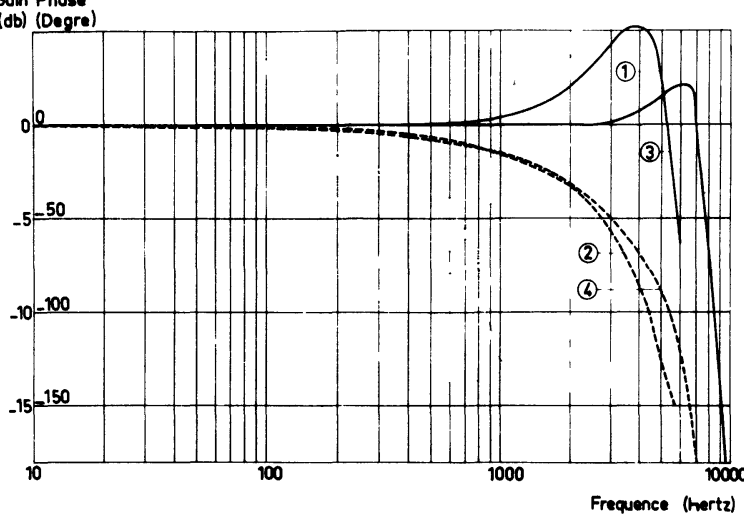

Fig. 7. - La réponse en fréquence de l'intégrateur. Courbes de gain et de phase en fonction de la fréquence : les courbes 1 et 2 sont relatives au galvanomètre (1); les courbes 3 et 4 au galvanomètre (2).

obtenu à la sortie de l'intégrateur quand le signal d'entrée est une impulsion très brève. Le tableau VII résume quelques résultats obtenus avec les deux galvanomètres. La figure 7 donne les courbes correspondantes.

4) LA CORRECTION EXTÉRIEURE. - Pour illustrer le fonctionnement du correcteur nous traiterons quelques exemples. Nous avons d'abord choisi l'intégrateur fonctionnant avec le galvanomètre (2). La constante de temps $\theta$ avait pour valeur $10^{-7}$ seconde. Nous avons comparé les réponses en fréquence de l'intégrateur avant et après correction. Le tableau VIII indique les valeurs respectives des fréquences $f_{0}, f_{1}$ et $f_{2}$ déjà définies, ainsi que le temps de montée d'un échelon de tension.

$\begin{array}{lcc} & \text { TABleaU VIII } & \\ & \text { Avant correction } & \text { Après correction } \\ f_{0}(\mathrm{~Hz}) & 7900 & 10000 \\ f_{1}(\mathrm{~Hz}) & 4850 & 8000 \\ f_{2}(\mathrm{~Hz}) & 1100 & 6750 \\ \text { Temps de monté } & 100 & 60\end{array}$

On constate sur cet exemple que le correcteur permet de diminuer le déphasage dans une très large bande de fréquences. La figure 8 compare les courbes de réponse avant et après correction. On constate que l'intégrateur corrigé déphase de moins de $10^{\circ}$ jusqu'à 4500 hertz. On voit également que le système corrigé présente une plus grande surtension que le système non
$\underset{(H z)}{f_{0}}$
$f_{1}$
$f_{2}$
Temps
( $\mathrm{Hz})$
$(\mathrm{Hz})$
(Hz)
de montée

$\mu \mathrm{s}$

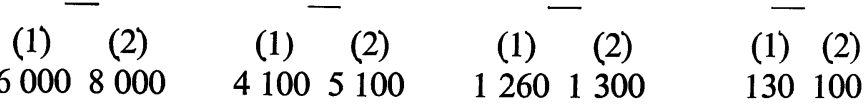

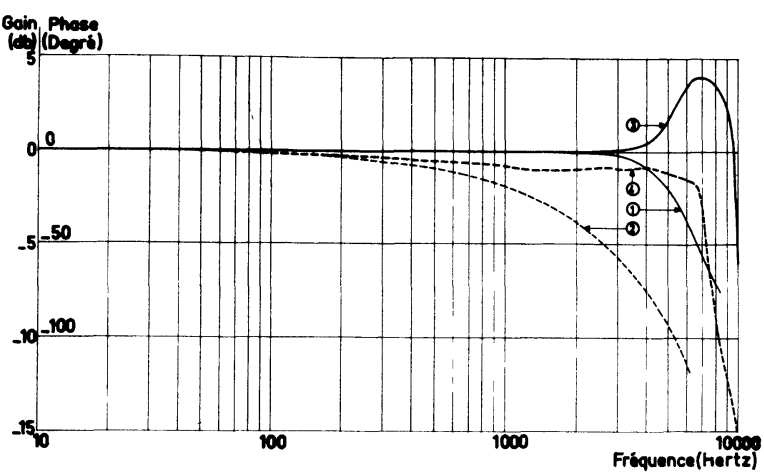

FIG. 8. - L'effet de la correction extérieure. 1 et 2 : courbes de gain et de phase, en fonction de la fréquence, avant la correction; 3 et 4 : mêmes courbes après correction.

corrigé, comme le montre un calcul que nous n'avons pas reproduit ici.

Le correcteur donne également des résultats très satisfaisants avec l'amplificateur linéaire. Le tableau IX montre les résultats obtenus au gain $10^{4}$ avec chacun des galvanomètres.

TABLEAU IX

Avant correction Après correction

$\begin{array}{llrr}f_{0}(\mathrm{~Hz}) & (1) & 1400 & 1860 \\ & (2) & 3980 & 5200 \\ f_{1}(\mathrm{~Hz}) & (1) & 780 & 1220 \\ & (2) & 2225 & 3460 \\ f_{2}(\mathrm{~Hz}) & (1) & 160 & 950 \\ & (2) & 510 & 2820\end{array}$

On constate ici aussi que le correcteur élargit la bande passante et diminue très notablement les déphasages. De nombreux essais ont montré qu'on peut réduire le déphasage à 10 ou $20^{\circ}$ jusqu'à la fréquence où il valait $135^{\circ}$ auparavant. Ce résultat tend à confirmer le fait qu'on trouve théoriquement une fonction de transfert du troisième ordre pour représenter le système bouclé, du moins dans une bande de fréquences de plusieurs kilohertz. Il est également intéressant de remarquer que ce procédé est très souple, car nous disposons de paramètres de réglage qui sont indépendants. Il est possible de régler presque à volonté la forme de la réponse en fréquence. On peut, en particulier, soit obtenir une courbe de gain pratiquement plate depuis le continu jusqu'à une fréquence aussi élevée que possible, soit minimiser le déphasage dans une bande de fréquence donnée. 
V. Conclusion. - Il est intéressant de comparer l'appareil que nous avons obtenu avec les maillons purement électroniques.

L'amplificateur linéaire corrigé passe à moins de $20^{\circ}$ de déphasage environ $1000 \mathrm{~Hz}$ au gain $10^{4}$, $700 \mathrm{~Hz}$ au gain $10^{5}$ et $400 \mathrm{~Hz}$ au gain $10^{6}$. Les produits gain-bande passante correspondants valent respectivement $10^{7}, 7 \times 10^{7}$ et $4 \times 10^{8}$, encore sont-ils définis de façon très pessimiste, car la courbe de gain est parfaitement plate jusqu'aux fréquences indiquées cidessus. Les meilleurs amplificateurs opérationnels sont caractérisés par un produit gain-bande égal à $10^{8}\left({ }^{1}\right)$. L'intérêt de l'amplificateur galvanométrique apparaît dès qu'il devient nécessaire d'utiliser des gains élevés.

En ce qui concerne l'intégrateur, on peut le comparer avec deux des appareils actuels les plus performants ; les nanovolmètres "Astrodata " modèle $121 \mathrm{Z}$ et "Keithley» modèle 148. Ce sont des amplificateurs linéaires ; mais on peut les prendre comme base de

(1) Il s'agit ici de la bande passante au gain unité. comparaison car l'intégration à leur niveau de sortie ne pose pas de problème particulier. Le premier est caractérisé par une bande passante de $100 \mathrm{~Hz}$ et une dérive intrinsèque de 50 nanovolts par heure. Le second, pour 10 nanovolts pleine échelle a un temps de montée en signal carré de 5 secondes et une dérive intrinsèque de quelques nanovolts par heure. L'appareil que nous avons réalisé possède à la fois une bien meilleure stabilité et un temps de montée très inférieur. Cet appareil doit permettre de résoudre de nombreux problèmes relatifs à la mesure des très faibles tensions continues; il nous a permis de construire un fluxmètre très sensible avec lequel nous pouvons envisager de faire des mesures dans des domaines jusqu'à présent inaccessibles.

Remerciements. - Les nombreuses discussions que nous avons eues avec Messieurs P. Brissonneau, J. Max et B. Cazenave ont été très fructueuses. Nous leur savons gré de l'intérêt qu'ils ont porté à cette étude. Par ailleurs, Monsieur J. Max a bien voulu accueillir dans son groupe Monsieur Torlet, technicien chargé de la réalisation de l'électronique, nous l'en remercions vivement.

\section{Bibliographie}

[1] EdgaR (R. F.), Electrical Engineering, 1937, 56, 805.

[2] Dicke (R. H.), Rev. Sci. Instrum., 1948, 19, 533.

[3] Cioffi (P. P.), Rev. Sci. Instrum., 1950, 21, 625.

[4] Cioffi (P. P.), Rev. Sci. Instrum., 1954, 25, 624.

[5] Berge (R. I.) et GuderJahn (C. A.), Electronics, 1954, 27, 147.

[6] KAPITZA (S. P.), Zhurnal Tekhnichvhoy fiziki, 1955, 25, 1307.

[7] LEROND (P.) et ThULIN (§̊.), Journal of Sci. Instrum., $1959,36,388$.

[8] Bockemuehl (R. R.) et Wood (P. W.), Electronics, 1960, 32, 70 .
[9] Grivet (P.), Sauzade (M.) et Stefant (R.), Rev. Gén. de l'Electricité, 1961, 70, 317.

[10] Max (J.) et Chevalier (R.), J. Phys. Appl., 1964, 25, $90 \mathrm{~A}$.

[11] Cazenave (B.), Brevet français, $\mathrm{n}^{\circ}$ 1-265-274 du 23/5/64. Mesures, octobre 1964.

[12] Brissonneau (P.) et Pechart (R.), Brissonneau (P.), Communication personnelle.

[13] Milatz (J. M. W.) et Wapstra (A. H.), Physica XIX, 1953, 166.

[14] KAPFer (E.), Archiv. für Technisches Messen, 1966, 364. 